\title{
Evaluation of Chromium and Selenium in Serum of Female Patients with Breast Tumor
}

\author{
Farhan, AR \\ Medical Technical Institute, AL- Mansur Foundation of Technical Education, Baghdad, Iraq,
}

\begin{abstract}
This is an explorative study, rarely conducted in Iraq relied on sample of women with breast tumors to measure the possible contribution of chromium $(\mathrm{Cr})$ and selenium(Se)values in the serum and hair to breast tumor genesis. Serum and hair samples were taken from 59 females with age from 25 to 60 years. Thirty four females with breast tumor were grouped according to the type of tumor into two groups (malignant: 20 and benign: 14) and twenty five were apparently healthy. The result demonstrated that scalp hair of patients with malignant breast cancer were significant increase of hair content of $\mathrm{Cr}$ with significant decrease of hair Se, compared to benign and healthy controls. The levels of serum Se were significantly decreasedin both malignant and benign groups, compared to controls. A Significant decrease in hair contents of Se was seen in malignant tumor groups, compared to controls. Non-significant increase in the level of serum $\mathrm{Cr}$ in the malignant and benigngroups, compared to controls.
\end{abstract}

Keywords: Trace elements, Hair, Serum, Breast Tumors.

\section{Introduction}

Breast cancer is a common tumor in females worldwide (1). Breast tumor was described as either benign or malignant. Breast is constantly responding to the changes in hormonal, genetic, nutritional, psychological and environmental stimuli that cause continual cellular changes (2). Element biotransformation in tumor tissue might help to elucidate the potential of element to tumor and to decide whether these elements could be used as additional biochemical markers for the diagnosis and prognosis of tumor development (3).Many of essential trace elements are required for normal and tumor growth.Assessment of body status for a particular element is extremely difficult, for years there has been considerable controversy over hair mineral analysis(4). Most of the elements enter the human body mainly by the circulating blood and leave mainly through urine and feces. Other mechanisms of elimination are secretion through sweat, milk, menses or semen or accumulation in hair, nails and teeth(5). Although hair is the most convenient tissue for analyzing minerals, only a limited number of elements in the hair accurately reflects intracellular or extracellular specific cell status, such as leukocytes (6). Hair has a number of advantages compared to serum, it is easily collected, stable on storage and has a higher concentration of elements, but many individuals and external factors can influence element levels $(7,8)$. Any Comparative study, attempting to define accuracy, is seriously, flawed and can yield only disparate and inconclusive results(9). The accepted means of assessing the validity of any laboratory test, including hair element analysis, is by measuring its reproducibility and accuracy in comparison to certified standards(10). Hair analysis is best viewed as an important clinical tool that helps provide clinical guidance in conjunction with each patient unique counseling of presenting symptoms, lifestyle and health risks. When used in this way, we believe that hair element analysis conveys clinically reliable andvaluable information for healthcare providers $(11,12)$

Human hair possesses $85 \%$ protein, $15 \%$ water and small amount of lipids and inorganic materials. The mineral content of the hair is $0.25 \%-0.95 \%$ in a dry ash basis (13). Unlike other body tissues, hair is a metabolic end product that incorporates elements into its matrix, it is affixed permanently and becomes difficult to remove through normal washing because of the exposure of hair follicles to the blood supply during growth, element concentration of the hair reflects concentration in the body over thetime(14). For some elements, concentration can be more than two hundred times higher in hair than in blood or urine (15). This increased concentration offers greater sensitivity and precision for the detection and quantification of many trace elements in the sample. In addition, hair unlike most other biological tissues is stable. It doesn't require thermal or chemical preservation during storage.Many studies had documented significant relationship between element concentration in hair and other tissues and fluids (16). These studies showed that using hair analysis to detect variations of elements in the body establishedthe relationship between their concentration and disease(17). Hair element levels have been correlated not only with active symptoms on disease states, but with the further development of disease condition, thus suggesting heir powerful preventive potential in serving as predicting riskmarker (18).

There are few studies concerning the determination of element levels in blood, urine and hair of breast cancer patients, most of them deal with $\mathrm{Cr}, \mathrm{Zn}, \mathrm{Cu}$ and $\mathrm{Se}$ because of the role these elements in the antioxidant 
defense system of the organism. Sub toxic level of $\mathrm{Cr}$ as ( $\mathrm{Cr}$ III nitrate) and Se as Sodium-Selenite are found in water supplies. $\mathrm{Cr}$ counteracts the inhibitory effect of selenium on tumor development in a dose dependent manner, shortened the tumor latency period and accelerated tumor growth rate, exposure to $\mathrm{Cr}$ also altered the levels of Se in the liver and kidney of the mice indicating that $\mathrm{Cr}$ interacts with $\mathrm{Se}$ and affects its organ distribution. Chromium must be added to the list of selenium-antagonistic elements that weaken or abolish anti tumorigenic effect of Selenium $(19,20)$. The presence of these elements in food must be taken into account when estimating the optimal dose of supplemental Selenium for cancer risk reduction (20). Chromium is an essential trace element, body content is between $0.4-6 \mathrm{mg}$, older people usually have lower levels. There is a wide geographical variation in $\mathrm{Cr}$ levels and population studies suggest that the incidence of diabetes and heart disease is lower in areas where $\mathrm{Cr}$ intakes are relatively high. $\mathrm{Cr}$ is essential for normal sugar metabolism. It is a component in glucose tolerance factor (GTF) which works with insulin to push more glucose into cells where it can be used co generates energy(21). Selenium is also an essential trace element in human and animals, it is a component of glutathione peroxidase (GSH-PX) which has a cellular antioxidant function (22) and appears to play a key role in reducing the risk of some cancers, cardiovascular diseases, diabetes and arthritis $(23,24)$. Low Se levels in blood, urine and hair, have been significantly observed in patients with breast cancer (25).

\section{Materials And Methods}

\section{Collection of hair and serum samples:}

Hair sampleswere taken from occipital region of the head, since this area is less visible and less contaminated(26). We used a sample of fifty nine for analysis, based on the development of an advanced methodology, which meets lower required amount to dilute the sample up to thespecified volume (27). All the patients and controls were fasted during sampling and responded freely to questionnaires and clinical investigations. Exclusionsincluded pregnant or lading women, women on hormonal therapy, with inflammatory conditions, taking supplements containing Se; women with diabetes mellitus, hypertension, hepatitis, and women taking any drugs effecting mineral measurement.

\section{Subjects}

A total of fifty nine women with diagnosed with breast tumor, aged (25-60) years have been examined and formed the initial study group, who had been admitted in different hospitals in Baghdad. The breast tumors patients were subdivided into two groups:-Group 1: consisted of 14 patients with benign tumors, Group2: Consisted of 20 patients with malignant tumors. All patients were fully informed about the study and informed consents were obtained from all subjects. About twenty five healthy women with ages matching to the patient groups were included in this study as the control group.

\section{Measurement of minerals in hair and Serum:}

Digestion \& preparation of hair sample:Hair samples were digested according to the procedure of Alan Cuwy (1979) by acid (28). Washing of the hair sample is critical to remove loosely bound, exogenous metal containing materials. Shampoo, conditioner, rinses, light, sweating and air pollution generally do not significantly affect hair readings. Most people wash their hair frequently, most hair products do not contain many minerals that remain in the hair, therefore the test was notaffected. Most contaminants do not remain within the hair. However, swimming in pools can raise sodium \& copper levels. Heavy sweating immediately before cutting the sample can raise sodium \& potassium readings. Grecian formula hair dye contain lead, they will elevate the lead level, (and should be avoided). Some shampoos can elevate the Zinc level. Other shampoos can elevate the Se level. Asking the patients what products are on. Their hair will usually be sufficient to rule out abnormal readings due to the hair products $(10,29)$. Bleach or other chemicals used in permanents may have some effect on hair readings.Hair samples were taken before having permanent or bleaching, after washing the hair 4-5 times after these treatments before having a hair analysis however, if a person is very ill, a sample can be taken at any time. Hair submitted for analysis is cut into very short pieces, samples are rinsed with acetone and allowed to drain, each sample is washed twice with detergent which has been shown more effective than other cleaning agent for removing contaminants safely without affecting internal elemental composition of the hair matrix (29).However the use of such washing methods prior to elemental analysis removes minute quantities of elements from the hair strands. This degree of loss is dependent on the washing method employed $(16,19)$, this washing is followed by rinsing with deionized water, two times with acetone and samples were then dried at approximately 75 degree Celsius(10). Minerals in serum and hair samples were estimated by Atomic absorption technique.

\section{Statistical Analysis}

The results were presented as mean \pm standard deviation (SD) and range, using SPSS package, using students t-test considering $\mathrm{P}<0.05$ as the lowest limit of Significance. 


\section{Result and discussion}

A Significant decrease was observed in serum Se value in malignant $(0.29 \pm 0.19 \mu \mathrm{mol} \vee 1)$ and benign $(0.35 \pm 0.155 \mu \mathrm{mol} l \mathrm{l})$ groups, compared to healthy controls $(2.5 \pm 1.4 \mu \mathrm{moll})$, as shown in Table 1 . It was previously reported that a considerable negative correlation between hair Se content and the type of tumor was found. In females with breast cancer, the Se levels in serum, urine and hair were significantly lower than control(25).Non significant difference was found between malignant and benign groups for serum Se level. As illustrated in Table 2, hair Se in malignant breast tumor patients showed a significant decrease, compared to control groups, and benign groups and non Significant difference in hair Se between controls and benign breast tumor patients.Xue, etal. (30), found a reduction in serum Se in patients with ovarian cancer, they considered the decrease in serum Se as a useful parameter for the diagnosis and treatment of those patients and considered this reduction as a result of protective migration of Se from blood to the cancer tissue,so this explained the association between oxidative stress and changes of trace elements in breast cancer patients. These results agree with the finding of Sieja (31).

$\mathrm{Cr}$ is an essential trace mineral for normal sugar metabolism which helps insulin to move glucose into cells. The presence of this mineral in food must be optimized for breast cancer risk reduction (21). As illustrated in Table 3,there was non significant differences in serum $\mathrm{Cr}$ between control, benign and malignant breast tumor patients while, a statistically significant increase in hair content in malignant tumor patients, compared to control and benign.No significant differences in hair $\mathrm{Cr}$ between controls and benign breast tumor patients $(p>0.05)$ as shown in Table 4.These results were in agreement with some reportsshowing that breast and other major forms of cancer are inversely affected with the dietary Se intake, but directly correlated with the estimated intake of chromium and other Se antagonistic elements. The presence of these elements in food must be taken into account when estimating the optimal dose of supplementary Se for cancer risk reduction (32).Hair $\mathrm{Cr}$ content is notably higher in cancer patients, which may lead to a number of physiological disorders. More recent studies established that dietary $\mathrm{Cr}$ acts as a Se antagonist. Seshowed anti carcinogenic properties in numerous animal models and there is evidence that cancer mortalities in different countries are inversely associated with the dietary intakes of this element (27). However, Se interacts with some toxic heavy metals during detoxification processes, resulting in inactivation of Se, that by time, produces a state of akin (Se deficiency) $(20,31)$.Our results about Se and $\mathrm{Cr}$ variations agreed with previously reported results (33), that showed that scalp hair of patients with oncological mammary pathology are characterized by a significant decrease of the concentration of Se and Zinc and by the increase of Cr. This means that, the study of trace element content allows controlling the balance of trace elements incorporate into the structure of antioxidant enzymes responsible for antitumor protection. Direct evidence for interactions between $\mathrm{Se}$ and $\mathrm{Cr}$ in humans is still scant but some has become available supporting the results of the original correlational calculations. Thus, blood Se levels in Polish tannery workers exposed to $\mathrm{Cr}$, were significantly lower than in unexposed controls(20). Lower Se and elevated $\mathrm{Cr}, \mathrm{Zn}$ and $\mathrm{Hg}$ concentrations were observed in blood of Indian breast cancer patients compared to cancer-free controls (34).

Table 1: Serum Selenium level (Mean \pm SD and range) of patients with benign and malignant breast tumors and normal controls:

\begin{tabular}{|l|c|c|c|}
\hline Serum Se $(\boldsymbol{\mu m o l} \mathbf{L})$ & Controls & reast Benign Tumor Patients & $\begin{array}{c}\text { Breast Malignant Tumor } \\
\text { Patients }\end{array}$ \\
\hline Sample size & 25 & 14 & 20 \\
\hline Mean \pm SD & $2.5 \pm 1.4$ & $0.35 \pm 0.155$ & $0.29 \pm 0.19$ \\
\hline Range & $1.1-4$ & $0.2-0.45$ & $0.14-0.4$ \\
\hline P Value & & 0.00011 & NS $* *$ \\
\hline
\end{tabular}

** Comparison ofmalignant to benign breast tumor patient.

Table 2: Hair Selenium level (mean \pm SD) and range of patients with benign and malignant breast tumors and normal controls:

\begin{tabular}{|c|c|c|c|}
\hline Hair Se (pg)g) & Controls & Breast Benign Tumor Patients & Breast Malignant Tumor Patients \\
\hline Sample size & 25 & 14 & 20 \\
\hline Mean \pm SD & $0.94 \pm 036$ & $0.89 \pm 0.32$ & $0.77 \pm 0.23$ \\
\hline Range & $0.5-1.5$ & $0.6-1.35$ & $0.5-1.0$ \\
\hline P value & & $\mathrm{NS}^{*}$ & $\mathrm{P}<0.003^{* *}, \mathrm{P}<0.01^{* * *}$ \\
\hline
\end{tabular}

*For comparison between Benign breast tumor patients to controls.** For comparison between malignant breast tumor patients to controls. ${ }^{* * *}$ For comparison between malignant breast tumor patients controls \&Benign breast tumor patients. 
Table 3: serum Chromium level (mean \pm SD) and ranges in patients with Benign \& malignant breast tumors compared to normal controls:

\begin{tabular}{|c|c|c|c|}
\hline Serum Cr $(\boldsymbol{\mu m o l} \backslash \mathbf{L})$ & Controls & Benign & Malignant \\
\hline Sample size & 25 & 14 & 20 \\
\hline Mean \pm SD & $0.011 \pm 0.0095$ & $0.015 \pm 0.009$ & $0.015 \pm 0.005$ \\
\hline Range & $0.001-0.02$ & $0.0061-0024$ & $0.0055-0.02$ \\
\hline P Values & & NS* & NS**, NS*** \\
\hline
\end{tabular}

*For comparison between Benign breast tumor patients \& controls. **For comparison between Malignant breast tumor patients\& controls. *** For comparison between Malignant breast tumor patients \& benign breast tumor patients.

Table 4: Hair Chromium level (mean \pm SD and Range) of patients with benign \& malignant breast tumor and normal controls:

\begin{tabular}{|c|c|c|c|}
\hline Hair $\mathbf{C r} \boldsymbol{\mu g} \backslash \mathbf{g}$ & controls & Benign & Malignant \\
\hline Sample size & 25 & 14 & 20 \\
\hline Mean \pm SD & $2.4 \pm 0.4$ & $2.5 \pm 0.39$ & $4.2 \pm 0.8$ \\
\hline Range & $2.0-2.8$ & $2.11-2.89$ & $3.4-5$ \\
\hline P values & & NS & $\mathrm{p}<0.001^{* *}, \mathrm{p}<0.00^{* * *}$ \\
\hline
\end{tabular}

* For comparison between Benign breast tumor patients \& controls. ** For comparison between Benign breast tumor patients \& controls. ${ }^{* *}$ For comparison between Malignant breast tumor patients\& controls.

\section{Conclusion And Recommendation}

The study necessitates the evaluation of both $\mathrm{Cr}$ and Se figures in serum, hair and urine of hair fall suffering patients and to be prescribed while all treatment modalities for breast cancer patients to safeguard therapeutic response.

\section{References}

[1] AnthorngS.E,;Eugene, B.;Kurt. JI: Harrison's principles of Internal medicine $4^{\text {th }}$ ed. new York (1998).

[2] Proth. C.M .pathophysiology $4^{\text {lh }}$ ed .Philadelphia J.B, Lippi (1994).

[3] Gupta. S.K, Shuks V.K .Valoya M.P, Roys K and Grapts: Serum trace element and CulZn ratio in breast cancer patients, Surg. Oncol. 160:178-182 (1991).

[4] TimarJ;Roso E, Pakuskopper L.; and zinc inhibit liver metastasis of 3LL1-HU murine tumor cells . In. J. Med .2:105 (1999)

[5] G. V. Lyengar, W. E. Kolllmr/H.g.m. Bowen: (The elemental compostion of human tissue and body fluids), verlag chemic. Weinheim-New York (1978).

[6] A text book of Natural Medicine, Stephen Markus MD; Mineral status Evaluation (1992).

[7] Murray, R.K., Granner, D, Mayes .P.A and Rod well, V.W; Harpers Biochemistry $25^{\text {th }}$ edition (2002).

[8] Majewska U, Braziewiez J., Banas, Kubala - kuks. A; An elemental Correlation study in cancerous breast cancer tissue by total reflection X-ray fluorescence Boil trace Elem. Res, Go(1-2); 91 -100 (1997).

[9] Glad MJ, Kaminksy M.V: Accuracy of hair mineral analysis (letter).JAM ,285 (12): 1577 (2001).

[10] Seidets, S. Keroulizer, R. Smith, D.: Assessment of commercial laboratories performing hair minerals analysis JAMA, $285: 67-72$. (2001).

[11] Manson P, Zlotkin S, Hair analysis, a critical review .Can. med Assoc. J,133:186- 8,(1985).

[12] Rayn DE, Holzbecher.J.StuartD.C.Trace elements in Scalp hair of person with multiple Sclerosis\&normal individuals.Clin.chm,24:1996-2000

[13] Katzs A. Chatt A.; Hair analysis application in the biomedical and environmental sciences new York VC H publisher (1988).

[14] Mephillips NA, Strang J, Baranes TRE; Hair analysis, new laboratory ability to. test for substance. Use Br. J. psych ; 173:287290(1998).

[15] Chatropadhyay A, Roberts ; TM, Jervis RE; Scalp hair as a monitor of community exposure to lead Arch. Environ. Health , 32(5)( 1977).

[16] Hachet ,D.S. and siggia ,S.; Environmental analysis, academic press newyouk.p253.( 1977).

[17] Goulle J.P., Kinitic P.; A new tool for biological study in hair analysis, value in medical practical practice (French) .Rev. Med Inten.17 (10): 82b-35,(1996).

[18] Strain W.H., Steodmanl T., Lunkau .C.A., Berliner W. P.; deficiency in man ,J. lab. clin .med .; 681 (2): 244,(1966).

[19] Borella P. Bargellini A, Caselgrndi E and Piecinini L.; Observation on the we of plasma, hair and tissue to evaluate trace element status in cancer .J elem. Med .Bio .1, LL: 162-165,(1997).

[20] ScharanerG.N;Ineractive ,effecls of selenium and Chromium on mammary tumor ; Department of Biochemistry' University of California; Bidogical Trace Elements Research Institute, Vol. 109 NO.3,281-292 (2006).

[21] The vitamin update Bookman media pty. ltd.; 36093085 51,(2011).

[22] Barceloux Dg; Selenium J.Toxicalclin .Toxial; 37(2): 145-72 (1999).

[23] Abid F. M., Hamach, Ahmed A. Shanshal ,H.A Hameed; Trace Elements for good health Supplementation and Varian diseases Iraqi Atomic energy commission AL- Naharin University (2002).

[24] welliness (letter), the news letter, of nutrition, fitness and self care from the school of public health volume 18 , (2000).

[25] Bratakes M.S., Vouterakos T.B., Ionncu P.V.; Selenium status of cancer in Greece Sci. total Environ, Mar .92 :207 -22 (1990).

[26] LotherThomas, M.D.I ${ }^{\mathrm{sl}}$ ed; clinical laboratory Diagnostic use and assessment of clinical laboratory results (1998).

[27] Gramtor E Passvvaler RA.; Trace element, hair analysis and nutrition, New Cannan .CT: Keats publishing Inc .(1993).

[28] Alan curry M.A; poison detection in human organs $3^{\text {ed }}$ ed .p 124-125 (1979).

[29] SenJ,DasChandhuri AB; Brief communication ; choise of washing method of hair Samples for trace elements analysis in 
environmental studies ,Am J.phs . AnthropolJul;1 IS (3). 289-91, (2001).

[30] XUE ,FX ,Z hang sw.; selenium concentration in serum, hair and tumors tissue from patients with ovarian tumors, znanghuafuchan ;26(5):290-2:(1991).

[31] Siegak ; Selenium deficiency in women with ovarian cancer undergoing chemotherapy and the influence of supplementation with this microelement on biochemistry parameters , pharamzi Jul; 35(7): 473-6(1998).

[32] Kilic E., Scraymen P., Biol. .trace Elem Res .; Department , of Biochemistry clinical Biochemistry , Erciyes university 38039 Kayseni, Turkey ; 102 (1-3) 19- 25 .(2004).

[33] YuriyKolmogorrow, Valautinakovaleva, Alexander Gonchar ; Analysis of trace elements in scalp hair of healthy people , hyperplasia and breast cancer patients ; Elsevier; 448(457-460),(2000).

[34] Vsingh and A. N. Garg, Trace element correlations in the blood of Indian women with breast cancer, Biol. TraceElem Res. 64, 237245 (1998). 8 